\title{
El sistema educativo cubano en los noventa*
}

\section{O neida Álvarez Figueroa}

Centro de Investigaciones de Economía Internacional.

U niversidad de La H abana. Cuba

Fecha de recepción: noviembre de 1995

\section{Resumen}

La estrategia cubana de desarrollo ha concedido especial atención al sistema educativo como uno de los ejes para lograr la modernización de la estructura económica, reduciendo las desigual dades sociales. D esde esta perspectiva, el presente artículo ofrece un panorama general del sistema educativo, comenzando por un análisis histórico en el que se trata de la etapa colonial española, del impacto de la ocupación norteamericana, de la etapa prerrevolucionaria y del desarrollo de la educación a partir de 1959, poniendo mayor énfasis en el análisis del sistema educativo cubano durante la presente década. Para hacerlo, se estructura el trabajo en tres partes que analizan las características y retos principales de la enseñanza básica, media y superior respectivamente. Se presentan los principios del sistema, su estructura, organización, niveles logrados en los principales índices valorativos del sector y su dinámica, y se intenta señalar los principales nudos gordianos en la perspectiva del cercano siglo XXI.

Palabras clave: educación, sistema educativo, escuelas, universidad, personal docente, tasa de escolaridad, presupuesto educacional, enseñanza básica, alumnos, planes de estudios.

\begin{abstract}
Cuban educational system in the ninety's
C uban development strategy has centred in the educational system, in order to modernize the economic structure and reduce social inequalities. The article examines the historical evolution of Cuban educational system from Spanish colonization, the 1959 revolution to the present decade. It analizes the main aspects of primary, secondary and higher education, as well as the most important endeavour towards xxith Century.
\end{abstract}

Key words: Education, educational system, levels of education.

* Este artículo se confecciona con la valiosa colaboración del Sr. Emilio Álvarez Aguiar (mi padre), especialista jubilado de la Dirección Provincial de Educación de C iudad $\mathrm{H}$ abana, del M inisterio de Educación, que ha escrito un libro sobre el desarrollo dela educación en Cuba (inédito). 


\section{Sumario \\ Introducción El sistema educativo de Cuba \\ Breve esbozo histórico \\ en los noventa}

Consideraciones finales

\section{Introducción}

El presente trabajo ofrece, en apretada síntesis, un panorama general del sistema educativo cubano en la actualidad, precedido de un breve esbozo histórico. Para facilitar la exposición se ha estructurado en tres partes, que analizan las características y los retos principales de la enseñanza básica, el nivel medio y la educación superior, respectivamente.

El tema de la formación, recalificación y superación de los recursos humanos adquiere cada vez mayor importancia en la estrategia de desarrollo de Cuba, por constituir uno de los ejes centrales de las transformaciones sociales logradas, y una de las bases fundamentales para elevar la competitividad de su economía y posibilitar una efectiva reinserción en los mercados internacionales.

Por esas razones, y porque la mayoría de la población cubana se siente protagonista de esa obra, desde posiciones educadoras o como educandos, resulta difícil concebir un análisis del sistema educativo excepto de apasionados debates, interesantes comparaciones internacionales, juicios val orativos sobre los avances alcanzados, percepciones diversas en torno a su eficiencia, y múltiples juicios valorativos sobre los avances alcanzados, percepciones diversas en torno a su eficiencia, y múltiples consideraciones para su ulterior perfeccionamiento.

Sin embargo, una arista insuficientemente desarrollada entre los interesados en la comprensión y transformación del espacio educativo cubano es la sociología de la educación. Este artículo no podrá trascender esa limitación porque resulta muy escasa la información estadística actualizada para revelar todos los matices de la caracterización social de docentes y alumnos en los diferentes niveles de educación, aún reconociendo la necesidad y el interés de ese análisis y de investigaciones serias con dicho objetivo.

N os circunscribiremos a presentar, con la mayor objetividad posible, los principios del sistema, su estructura, organización, niveles logrados en los principales índices valorativos del sector, y su dinámica. Las consideraciones finales que se exponen no tienen la pretensión de realizar un balance 0 una evaluación general del sistema, aspecto éste que dejamos a los lectores, sino señalar sus principales nudos gordianos, de cara al cercano siglo XXI.

\section{B reve esbozo histórico}

Los primeros antecedentes del desarrollo de la educación en Cuba durante la etapa colonial española se encuentran en las leyes reales del siglo XVI que dispusieron la enseñanza de la lengua española y la doctrina cristiana a los indios 
sobrevivientes en el país y a los esclavos procedentes de África, labor que encomendaron, en lo fundamental, a los curas y doctrineros.

Cuba recibió la influencia del sistema educativo prevaleciente en España en esa época, que priorizaba la superación cultural de la nobleza, el clero y los funcionarios intermedios. Por esa razón se desarrollaron con mayor celeridad los niveles medio y superior, mientras que la enseñanza elemental quedó rezagada.

A partir de la segunda mitad del siglo XVI se fundaron en Cuba los primeros centros de enseñanza. Las fuentes informativas disponibles permiten afirmar que hubo una participación amplia de franciscanos y dominicos en esas actividades, y de cierto número de jesuitas. Puede afirmarse que estas acciones fueron los escasos destellos que recibió la colonia de un período de gran desarrollo cultural en España.

Un hito destacable en esa etapa colonial fue la fundación en 1728 de la Real y Pontificia Universidad de San Jerónimo, en el Convento de los D ominicos (hoy U niversidad de La H abana). Su claustro estaba integrado por sacerdotes y su núcleo básico era la Facultad de Filosofía y Letras. N o obstante la transcendencia de ese acontecimiento, las repercusiones de esta institución durante sus primeros años de vida fueron muy limitadas, por su organización clerical, su carácter elitista y sus métodos escolásticos.

A partir de la segunda mitad del siglo XVIII se comenzó a desarrollar la ense ñanza elemental y se difundieron las escuelas para niños, aún cuando desde antes diversos representantes de la I glesia católica enseñaban a los menores a leer, escribir y contar. En 1793 se constituyó la Sociedad Económica de Amigos del País, institución que estuvo dirigida en sus orígenes por el C apitán General de la Isla y se ha mantenido activa hasta hoy, asumiendo diferentes funciones y cumpliendo objetivos diversos a lo largo de este amplio período histórico.

Con el impulso de esa Sociedad continuó ampliándose muy lentamente la enseñanza elemental en Cuba, se puede afirmar que es a partir de entonces que se comienza a estructurar de manera incipiente este subsistema educativo. Su heterogeneidad iba desde escuelas fundadas por creyentes ricos hasta aquellas atendidas por hombres y mujeres muy humildes que utilizaban sus propios hogares para enseñar a los niños, sin recibir en la mayoría de las ocasiones remuneración alguna. Los cabildos 0 ayuntamientos también participaban en esta labor, pero debe destacarse que a fines de siglo el Estado prestaba muy escasa atención a la enseñanza, las condiciones materiales de la mayoría de los locales eran muy deficientes, se retribuía mal a los maestros y éstos carecían de posibilidades de superaración.

Según datos del censo de 1801, en la capital del país había 71 escuelas, y unos 2.000 niños inscritos. En el resto de la Isla la situación era más precaria, por lo que la lucha por la atención a la educación se mantuvo constante, encabezada por los miembros de la Sociedad Económica de Amigos del País. Esta institución contribuyó decisivamente al establecimiento de requisitos para el ejercicio de los maestros, la atención a la superación de los mismos y el control sobre la calidad de la enseñanza. 
En 1836 el número de escuelas ascendía en Cuba a 223 y a ellas asistían más de 13.000 niños, atendidos por unos 400 maestros autorizados para enseñar. Estos avances se lograron como resultado de las presiones por interesar al gobierno en la instrucción pública, y gracias al aporte de fondos particulares y de los municipios, pero todavía el $87 \%$ de la población escolar no tenía acceso a la enseñanzal.

En 1841 se recibe en el país una real orden que establecía la Primera Ley Escolar de Cuba. Ésta disponía la fundación de las escuelas de primera enseñanza que fuesen necesarias y la instrucción gratuita para los niños pobres², así como la nacionalización de la U niversidad de La H abana. Pero esta legislación tuvo poca repercusión en la práctica.

0 tras disposiciones aplicadas muy limitadamente fueron la promulgación de una nueva ley escolar en 1863 que establecía la enseñanza obligatoria desde los 6 hasta los 9 años de edad, la organización de la inspección escolar ${ }^{3}$ y la fundación de institutos de segunda enseñanza.

Reformas sucesivas a los planes de estudio y una exigua ampliación del sistema educativo, con la fundación de al gunas escuelas técnicas, de oficios, de pintura, para niños desvalidos, correctoras de conductas, etc. caracterizaron la segunda mitad del siglo xIx, pero al momento de la liberación de España la situación educacional de Cuba era muy crítica.

Para ilustrar lo anterior baste mencionar que, de acuerdo a las estadísticas recogidas por el censo de 1899, menos del $6 \%$ de los niños asistía a las escuelas, la tasa de analfabetismo era del $64 \%$, y superior entre la población negra. Se calcula un número de escuelas inferior a 330, unos 1.200 alumnos de institutos secundarios y una matrícula cercana a 400 en la U niversidad de La $\mathrm{H}$ abana, para una población total de 1,7 millones de habitantes.

D ebe destacarse como lo más relevante de esa época en la historiografía educacional las actividades de eminentes educadores como José Agustín Caballero, Félix Varela, José Antonio Saco, Tomás Romay, Francisco de Arango y Parreño, José de la Luz y Caballero, Álvaro Reynoso, Rafael M orales y Rafael $M$ aría de $M$ endive, entre otros, cuyas ideas contribuyeron de manera significativa a crear conciencia de la necesidad de cambios cual itativos en el sistema, transformaciones en los métodos pedagógicos, conveniencia de introducir nuevas disciplinas, etc. Con justicia debe recordarse el auspicio del obispo J osé D íaz de Espada (fundador del Seminario de San Carlos en 1773, que a partir de entonces devino escenario para el desarrollo de los más notables gestores de la educación y forjadores de la nacionalidad cubana) y Alejandro Ramírez (responsabilizado con la dirección de la Sección de Educación de la Sociedad Económica de Amigos del País).

1. N ota del editor. En España la situación no era muy distinta. En 1803 sólo sabía leer y escribir el 5,96\% de la población y hasta 1838, cuando el absolutismo ya ha desaparecido, no se siente la enseñanza como una necesidad pública.

2. N ota del editor. En España, en 1841, sólo sabían leer y escribir el 9,21\% de la población.

3. N ota del editor. En España, en 1857, se aprueba la llamada Ley M oyano (en alusión a su autor) que establece por primera vez la enseñanza obligatoria para los niños de 6 a 9 años. 
Es fácil comprender que las fuerzas principales que guiaban el sistema educativo de Cuba y los impulsores de los cambios más progresistas que se fue ron introduciendo paulatinamente eran intelectuales procedentes de los estratos más acomodados del país.

El período de la ocupación norteamericana en Cuba, iniciado en 1898, favoreció los avances en el sistema de enseñanza, y en el segundo semestre de 1890 el número de escuelas primarias ascendía a más de 3.300 (10 veces más que las disponibles el año anterior). Los escolares asistentes a clases sobrepasaron los 135.000; se incrementaron los textos, que eran traducciones de libros norteamericanos; se introdujeron nuevas asignaturas en los planes de estudios y se formaron gran número de maestros.

D espués de la instauración de la República de Cuba se mantuvo la influencia del sistema de enseñanza norteamericano, y en 1909 se aprobó la Primera Ley Escolar, que regulaba en lo fundamental la instrucción primaria.

Las escuel as públicas eran insuficientes numéricamente durante la época republicana y la calidad sufría la escasez de recursos destinados a la educación. En las zonas rurales la situación era aún más precaria. En general a la débil base inmobiliaria, de materiales docentes, libros y otros medios auxiliares se sumaba la escasa atención a la remuneración, estimulación y superación de los maestros. Pero, a pesar de esas limitaciones, esa enseñanza gratuita y laica contribuyó a la formación de varias generaciones de cubanos que, con muchos esfuerzos, accedieron a recibir el legado de un magisterio mayoritariamente honesto, abnegado y solidario, con gran vocación por su trabajo.

D urante esa época merecen también destacarse las profundas modificaciones que sufrió la enseñanza universitaria, la ampliación de los institutos secundarios y la constitución del M inisterio de Educación (en la década de 1940).

En la etapa prerrevolucionaria se estima que la población cubana ascendía a 6,8 millones de personas, y ocupaba el tercer lugar de América Latina por el nivel del Producto Interior Bruto per cápita, pero su sistema educativo presentaba los rasgos típicos de los países atrasados económicamente: los hijos de familias de ingresos medios y altos tenían acceso a centros docentes privados, mientras la mitad de los niños en edad escolar no asistía a la escuela primaria; la tasa de analfabetismo ascendía al 22,3\% (entre las edades de 10 a 49 años); el nivel promedio de escolarización entre los mayores de 15 años era de 3 grados, y 10.000 maestros estaban sin trabajo (casi la mitad de los existentes).

La enseñanza industrial y agropecuaria contaba apenas 25 centros, con matrículas limitadas y escasos recursos; los estudios de administración y comercio se real izaban en una decena de institutos; la enseñanza preuniversitaria de nivel medio sólo era accesible a minorías de jóvenes urbanos; y para la formación y superación de maestros se contaba sólo con 6 escuelas normales y 3 facultades de educación.

Al triunfo de la Revolución Cubana, en 1959, sucede un profundo proceso de transformación, ampliación y perfeccionamiento paulatino del sistema educativo, que tuvo sus primeros objetivos en solucionar la herencia neocolonial antes expuesta. Para ello se realizó una reforma integral de la enseñanza, se 
emprendió una campaña de alfabetización masiva; se abrieron miles de aulas de primaria en todo el país; se concibieron y desarrollaron planes emergentes de Formación de M aestros; se organizó la Superación O brera para los adultos subescolarizados; se inició la Formación de Instructores de Arte y Promotores Culturales; se puso en marcha el Plan N acional de Becas; se crearon los Institutos de Superación Educacional para la calificación y recalificación de maestros; se constituyeron instituciones pedagógicas especializadas en el cuidado y educación de los niños desde sus primeros meses de vida hasta su etapa escolar; se impulsó la Educación T écnica y Profesional; se realizaron importantes transformaciones en la enseñanza media superior y universitaria; etc.

D espués de la etapa inicial de trabajo destinada a resolver las grandes carencias, insuficiencias y deformaciones heredadas, y a partir de 1975, los esfuerzos principales para el desarrollo de la educación cubana han estado dirigidos al perfeccionamiento del sistema nacional, la consolidación de la formación y superación del claustro y a darle integralidad al sector, en armonía con los requerimientos del desarrollo económico y social del país, convirtiendo los centros educacionales en instituciones culturales de la comunidad.

\section{El sistema educativo de C uba en los noventa}

N o se puede hablar de ningún elemento del nivel de vida de Cuba en la actualidad sin enmarcarlo en el contexto económico-social general del país. A partir de la desintegración del sistema socialista mundial, Cuba ha atravesado una de las mayores crisis económicas de su historia, a lo cual ha contribuido el bloqueo norteamericano, agudizado durante los últimos años. Baste decir que en 1993 el Producto Interior Bruto (PIB) había descendido en un 35\% con relación a 1989, y el déficit del presupuesto estatal al canzaba una proporción del $33 \%$ del producto. Una referencia sintética del nivel de la economía cubana hoy la ofrece su producto per cápita, que en 1994 ascendió a 1.174 dólares aproximadamente.

Aún cuando la tendencia decreciente de la economía parece haberse revertido y en los dos últimos años se han logrado discretos crecimientos de 0,7 y $2,5 \%$, la caracterización y los retos del sistema educativo hay que situarlos en un escenario muy complejo. Simultáneamente el país está modificando su inserción económica internacional, ajustándose a una disponibilidad de recursos sustancialmente menor, y diseñando una reestructuración económico-social que posibilite cumplir los dos objetivos anteriores y superar deficiencias internas del modelo aplicado hasta la presente década, preservando las conquistas sociales logradas.

Este epígrafe no pretende realizar un examen exhaustivo de la educación cubana, sino resaltar sus rasgos más relevantes, que permitan evaluar el lugar y el papel del sector dentro de una estrategia general de desarrollo.

El sistema educativo de Cuba está integrado por varios subsistemas: preescolar; general, politécnico y laboral; educación especial; educación técnica y profesional; formación y perfeccionamiento del personal pedagógico, educación 
Tabla 1. M atrícula inicial. Escuelas y personal docente. Curso escolar 1958-1959.

\begin{tabular}{lccc}
\hline N ivel de educación & Matrícula & Personal docente & Escuelas \\
\hline Total general & 938.138 & 29.237 & 8.009 \\
Primaria $(*)$ & 717.417 & $17.355(\mathrm{D})$ & 7.567 \\
M edia total & 88.135 & 4.571 & 81 \\
- M edia general & 63.523 & 2.580 & 21 \\
- Formación de maestros & 9.023 & 724 & 20 \\
- Técnica profesional & 15.586 & 1.267 & 40 \\
Especial & $176(\mathrm{~A})$ & 77 & 2 \\
Adultos & $24.939(\mathrm{~A})$ & $964(\mathrm{~A})$ & 325 \\
Juvenil (Esc. oficios) & $-(\mathrm{B})$ & $-(\mathrm{B})$ & $-(\mathrm{B})$ \\
Superior & 15.609 (C) & $1.080(\mathrm{E})$ & 6 \\
O tros & 3.730 & 669 & 28 \\
\hline
\end{tabular}

Fuentes: Dirección de Censo y Estadística G eneral. M inisterio de Educación.

Notas: $\quad(*)$ Incluye Preescolar a 60 grado.

(A) Año 1956-1957. D ato no disponible 1958-1959.

(B) No existía la enseñanza.

(C) Año 1956-1957. N o funcionaron las universidades oficiales, por la conmoción política que vivía el país.

(D) No incluye el sector privado. Dato no disponible.

(E) Año 1959-1960. Series históricas N ‥ 1 - 1958-1970, M IN ED . D ato no disponible en el año 1956-1957, al que se refiere la matrícula.

superior y educación de adultos (ver tabla 1). Administrativa y metodológicamente el sistema está atendido por los ministerios de Educación y deEducación Superior. Los elementos estatégicos y de política educacional son analizados en la Asamblea N acional del Poder Popular (Parlamento Cubano).

A partir del triunfo de la Revolución Cubana el sistema educativo se ha regido por un conjunto de principios, que le confieren al Estado la dirección y ejecución de todas las formas de enseñanza, ofrecidas a la población gratuitamente desde el grado preescolar hasta la universidad. Estos servicios se extienden a las zonas más apartadas del país, y tienen acceso a ellos toda la población, sin distinción de raza, sexo, religión o procedencia social.

La obli gatori edad de la enseñanza alcanza hasta el noveno grado, y se combina la teoría con la práctica en el proceso docente educativo, conjugando el estudio y el trabajo, con el objetivo de preparar mejor al educando para la vida social y laboral.

La tabla 2 permite comprobar que la tasa bruta de escolarización asciende al $54,7 \%$, y es ligeramente más alta en las mujeres $(53,7 \%)$, que a su vez representan prácticamente la mitad $(49,6 \%)$ de la matrícula total. Si este indicador se cal cula sobre la base de la población entre 6 y 23 años, asciende a 63\%. El promedio de los 17 países en desarrollo clasificados por el PN U D como de «al to desarrollo humano» es de $66 \%$.

La tasa de alfabetismo de la población mayor de 10 años es de $96 \%$, muy superior a la de América Latina y el C aribe, que asciende al 86\%, como pro- 
Tabla 2. Estructura de matrícula en el sistema de educación formal, para una población de 10.963.000 habitantes. Año 1993.

\begin{tabular}{lcr}
\hline & \multicolumn{2}{c}{ Cantidades en miles } \\
Tipo de enseñanza & Alumnos/as & Alumnas \\
\hline Preescolar & 296,6 & 136,3 \\
General básica & $1.334,2$ & 654,3 \\
M edia & 404,0 & 207,5 \\
Especial & 57,2 & 18,7 \\
Adultos & 114,3 & 65,3 \\
Superior & 165,8 & 95,6 \\
Total & 2.372 .1 & 1.177 .7 \\
Población de 0-24 años de edad & $4.497,9$ & $2.195,0$ \\
\hline
\end{tabular}

medio. Este indicador está por encima del que exhiben los países en desarroIlo clasificados por el PN U D como de «alto desarrollo humano» y que en su conjunto alcanzan una proporción del $8 \%$ de analfabetismo.

La atención al proceso docente educativo en Cuba cuenta con 270.000 maestros y profesores, que trabajan en los diferentes niveles, o sea 41 habitantes por maestro, la tasa más al ta del mundo. D e ellos el $60 \%$ atiende la enseñanza preescolar y la básica (de primero a noveno grado, que es el ciclo obligatorio); el $20 \%$ se ocupa en el nivel medio y el $10 \%$ integra los claustros de la educación superior. A lo anterior debe añadirse un 10\% destinado a trabajar en la enseñanza para adultos (casi 8.000) y en la especial (15.000, aproximadamente).

Estos dos últimos subsistemas merecen un breve comentario, por el elemento cualitativo que aportan a la educación cubana.

La educación de adultos se inició posteriormente a la C ampaña de Alfabetización, para no dejar incompleta esa obra, creándose aulas nocturnas en escue las y en centros de trabajo encaminadas a facilitar que todo el que lo deseara pudiera alcanzar el sexto grado.

Posteriormente, la educación de adultos comenzó a impartir nuevos niveles de enseñanza: secundaria obrero-campesina y facultad obrero-campesina, completando así la educación desde el nivel primario al preuniversitario, con planes y programas de estudios perfeccionados de acuerdo a los intereses y a las características de los estudiantes adultos. Éste constituye, por tanto, un sistema paral elo de enseñanza al de la educación de niños y jóvenes.

Al lograrse en 1980 la escolarización de todos los trabajadores y amas de casas con sexto grado, se inició la incorporación masiva para la obtención del noveno grado a través de los cursos secundarios. En el año 1988 completaron este nivel todos los trabajadores y las amas de casa aptos, comenzando una nueva etapa en la superación de los trabajadores. Ésta incluye dos posibilidades para la continuación de estudios: en la enseñanza técnica y profesional (donde elevan su calificación técnica y adquieren un nivel escolar del grado 


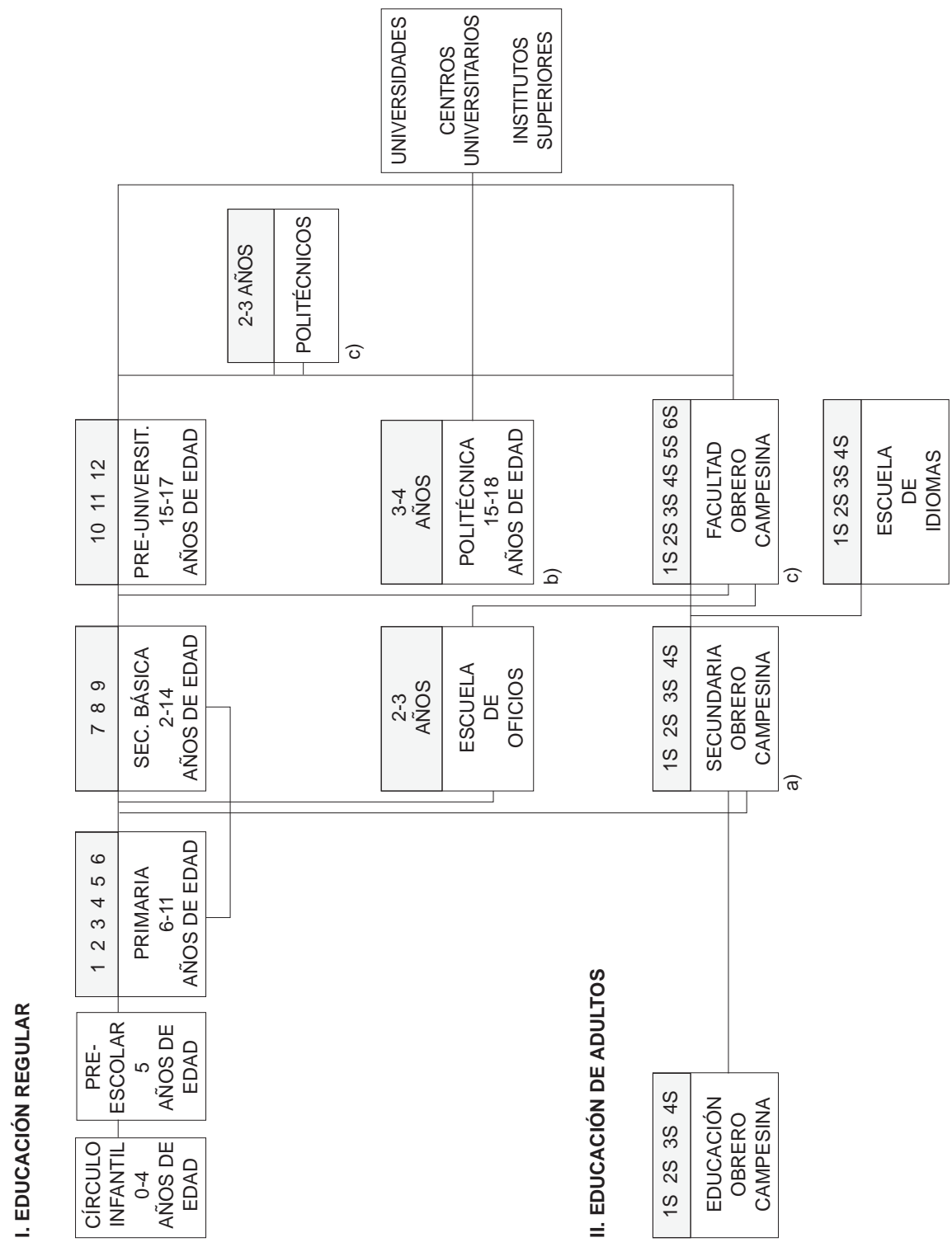

Figura 1. Estructura del sistema nacional de educación.

a) Los egresados de $\mathrm{SO} \mathrm{C}$ pueden también continuar estudios en centros politécnicos.

b) Pueden continuar estudios de educación superior desde sus puestos de trabajo y de acuerdo con su especialidad.

c) Pueden continuar estudios de educación superior desde su puesto de trabajo. 
doceavo al graduarse de técnicos medios), o en las facultades obrero-campesinas (en las que también al canzan el nivel medio superior). Ambos títulos capa citan para acceder a estudios superiores en las universidades, contándose por miles los que prosiguen dicho camino.

En 1993 la matrícula de este subsistema (algo más de 114.000 estudiantes) era casi el doble de la registrada en 1960 y cualitativamente diferente. Es muy significativo que la proporción de mujeres se el eva al $57 \%$.

La educación especial, por su parte, garantiza a los niños y jóvenes con deficiencias físicas, mental es y con trastornos de conducta una adecuada incorporación a la vida laboral como trabajadores.

En la educación especial se aplican planes de estudio, programas y libros de texto elaborados especialmente para cada uno de los diferentes tipos de deficiencias, tomando en cuenta las características propias de cada alumno. Este subsistema, casi inexistente hasta 1962, cuenta con 420 centros y 57.000 alumnos.

Los centros de diagnóstico y orientación, establecidos en todas las provincias del país, son los encargados de realizar el estudio previo de los niños y jóvenes que necesitan ingresar en escuelas especiales y brindan orientación y tratamiento a los que presentan dificultades en el aprendizaje. A los niños con severos impedimentos motores, se les garantiza sus estudios de nivel primario y medio con maestros ambulantes que asisten a sus hogares.

Además de los elementos cualitativos que aportan los servicios educacionales antes mencionados, hay un indicador que no puede obviarse al analizar el sector educacional. Se trata del nivel medio de escolaridad. Los datos que se presentan en la tabla 3 son el ocuentes.

Presupuesto educacional: Los gastos para sostener y desarrollar el sistema educacional en Cuba, en su totalidad, se sufragan a cargo del presupuesto estatal. Éstos han crecido sistemáticamente a partir del triunfo de la Revolución, pasando de 11 a 170 pesos per cápita entre 1958 y 19924. El gasto público en educación superaba los 1,7 miles de millones de pesos en 1990 y representaba el $12,8 \%$ del total de egresos del presupuesto. En la figura 2 se muestra la distribución del presupuesto educativo entre los diferentes subsistemas.

La magnitud de las cifras antes expuestas se comprende mejor si las referimos al Producto $\mathrm{N}$ acional Bruto y las comparamos con la evolución que esos indicadores han tenido en otros países (tabla 4).

No obstante la aguda crisis por la que ha atravesado Cuba en los últimos años, se han mantenido funcionando todas las escuelas, no se han afectado los índices promedio de promoción y no se ha quedado un solo maestro o profesor sin trabajo. Por supuesto, el proceso inversionista dedicado al sector se ha disminuido al mínimo indispensable y los gastos corrientes han sufrido reducciones.

4. Pesos comparables por su poder adquisitivo. 
Tabla 3. Promedio de años de escolaridad ( 25 años o más de edad). D atos del año 1992.

\begin{tabular}{lcll}
\hline Selección de países & Total & M ujeres & H ombres \\
\hline España & 6,9 & 6,6 & 7,1 \\
Uruguay & 8,1 & 8,6 & 7,7 \\
Argentina & 9,2 & 9,5 & 9,0 \\
Cuba & 8,0 & 8,1 & 7,9 \\
América Latina y Caribe & 5,4 & 5,2 & 5,5 \\
Países en desarrollo de & & & \\
$\quad$ «alto desarrollo humano» $\left.{ }^{*}\right)$ & 7,0 & 6,5 & 7,4 \\
Países en desarrollo de & & & \\
$\quad$ «medio desarrollo humano» $\left.{ }^{* *}\right)$ & 4,8 & 3,8 & 5,9 \\
Países industrializados & 10,0 & - & - \\
Países en desarrollo & 3,9 & - & - \\
Total mundial & 5,2 & - & - \\
\hline
\end{tabular}

Fuente: PNUD.

Índice sobre D esarrollo H umano, 1994. Fondo de Cultura Económica, 1994. (***)

Notas: $\quad(*) \quad$ Promedio de 17 países incluidos en esa clasificación por el PN U D en 1992.

(**) Promedio de 55 países incluidos en esa clasificacion por el PN U D en 1992.

(***) Índice de D esarrollo H umano (ID H ). M edición mediante indicadores representativos de tres dimensiones igualmente ponderadas del desarrollo humano: la longevidad (esperanza de vida al nacer), los conocimientos (logro educacional) y el ingreso per cápita.

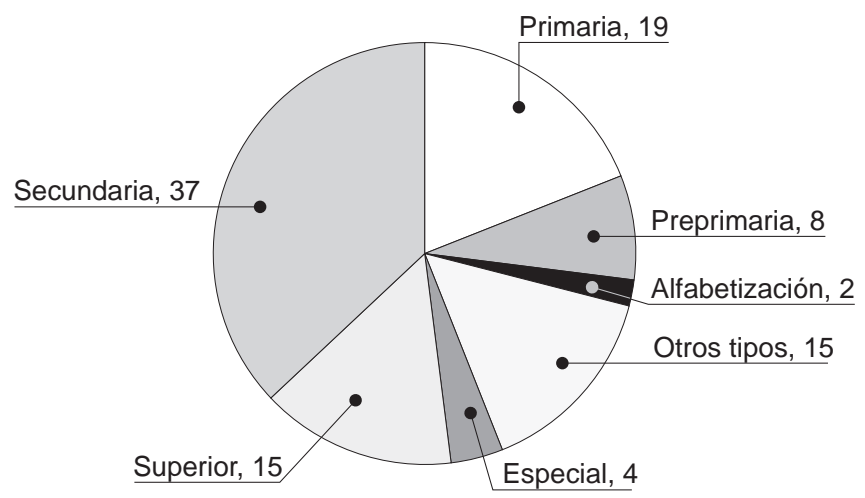

Figura 2. Presupuesto educativo (distribución porcentual). 
Tabla 4. Gasto público en educación (\% del PN B).

\begin{tabular}{lll} 
Países en desarrollo de & 1960 & 1990 \\
\cline { 2 - 3 } $\begin{array}{l}\text { «alto desarrollo humano» } \\
\text { Países en desarrollo de }\end{array}$ & 2,2 & 3,7 \\
«medio desarrollo humano» & 2,2 & 4,1 \\
Cuba & 5 & 6,6
\end{tabular}

Fuentes: PN U D . Informe sobre D esarrollo H umano, 1994. Fondo de Cultura Económica, 1994, p. 174.

Resulta difícil obviar que la calidad de la educación se ha afectado menos que proporcional a la contracción de recursos disponibles, debido a la creatividad y esfuerzo del personal docente, de las familias cubanas y a un fuerte movimiento de solidaridad internacional, que ha suplido la ausencia de financiamiento externo con donaciones de textos, materiales docentes, equipos y con asistencia técnica gratuita.

Afortunadamente, la metodología desarrollada por $\mathrm{N}$ aciones U nidas en su estudio sobre «D esarrollo H umano» nos permite evaluar comparativamente, con un solo indicador sintético, los resultados de los esfuerzos educacionales. En 1992 el índice calculado para Cuba en el indicador Logro educacional fue de 2,42 . Éste toma en consideración los niveles alcanzados en alfabetización de adultos y el promedio de años de escolaridad. Curiosamente este valor es idéntico al de España, que ocupa el lugar 23 (entre 173 países) por el índice de desarrollo humano. Las únicas naciones de América Latina que superan esa magnitud son U ruguay $(2,47)$ y Argentina $(2,53)$, aunque es destacable que se trata de países de mayor nivel económico, con Productos Interiores Brutos per cápita de 6.700 y 5.100 dólares, respectivamente (aproximadamente 5 veces el nivel de Cuba).

\section{Enseñanza básica}

Incluiremos aquí un breve análisis sobre el desarrollo del nivel preescolar, la enseñanza primaria (de primero a sexto grado) y la secundaria básica (de séptimo a noveno grado).

El subsistema preescolar representa el primer eslabón educacional, en el cual se sientan las bases para el desarrollo de la personalidad del niño, y se le prepara, paulatinamente, para su ingreso a la escuela.

Para llevar a cabo esta tarea se crearon los círculos infantiles en 1961, que son los centros de educación en los que se matriculan los niños de madres trabajadoras, desde 6 meses hasta 5 años de edad. $H$ ay dos tipos de círculos infantiles: los del plan regular y los del plan de educación especial para niños hipoacúsicos, con deficiencias en el desarrollo neuropsíquico e intelectual, etc.

En el país existen unos 900 círculos infantiles con una matrícula aproximada de 132.000 niños, atendidos por unas 8.000 educadoras (en su totali- 
dad mujeres). Estas se forman en los institutos superiores pedagógicos, en la carrera de Educación Preescolar.

El grado preescolar constituye un ciclo preparatorio de un año de duración para el comienzo de la educación primaria. Éste puede cursarse en los propios círculos infantiles o en las escuelas primarias, y tiene como objetivo fundamental preparar psicológicamente al menos para el aprendizaje escolar y propiciar su desarrollo físico, intelectual, moral y estético. A este grado ingre san los niños que han cumplido 5 años de edad. C asi 7.000 mujeres se desempeñan como maestras de preescolar en las escuelas.

Sin embargo, existe aún un alto porcentaje de niños, sobre todo en zonas rurales, a los que por la dispersión de sus viviendas no se les garantiza la posibilidad de recibir esta educación. Ello ha sugerido la realización de un plan remedial, gradualmente aplicado en las zonas rurales, con la colaboración de maestros y padres. Así se logra que todos los niños al iniciar el primer grado hayan recibido un mínimo de actividades preparatorias que faciliten su posterior aprovechamiento escolar. En la actualidad la situación económica del país ha limitado significativamente un amplio plan de desarrollo de nuevos Círculos Infantiles, por lo que la experiencia apuntada se ha comenzado a aplicar también en zonas urbanas. (Por vías no formales se preparan unos 200.000 niños menores de 4 años.)

En 1993 eran atendidos en las diversas modalidades de educación preescolar el $46 \%$ de niños menores de 5 años. Además de educadoras y maestras se suman a esta actividad las auxiliares pedagógicas (más de 15.000), promotores y personal de apoyo, hasta un total de 32,8 miles de ocupados, el $100 \%$ del sexo femenino.

Constituye una aspiración del sistema educativo cubano elevar las proporciones de niños menores de 4 años vinculados a la enseñanza preescolar, mediante su incorporación a instituciones o por vías no formales (hasta atender el 50\% en 1996 y el 80\% en el año 2000), con especial énfasis en los que no reciben estos beneficios en las zonas rurales.

La matrícula de la enseñanza primaria ascendió en 1993 a 983,5 miles de alumnos, representativos del 99\% de la población entre 6 y 12 años de edad. La asistencia a clases de estos niños es muy regular. Este nivel cuenta con más de 9.400 escuelas, y abarca de primero a sexto grado, divididos en dos ciclos. De primero a cuarto grado el niño transita con el mismo maestro; el quinto y sexto grado se dividen en dos áreas: ciencias y letras, impartidas por dos maestros especializados.

La enseñanza primaria representa hoy el $41 \%$ de la matrícula total del sistema educacional. Estas escuelas tienen diferentes tipos de régimen: externo, seminterno e interno. Los externos pueden ser de una sola sesión o de doble sesión.

Los niños externos almuerzan en sus hogares; los seminternos, desde 1960 almorzaban gratuitamente, hasta el inicio del curso 1994-1995 en que se estableció una tarifa de 7 pesos mensuales 5 . El internado para la educación pri-

5. El nivel de salario medio es de 200 pesos, aproximadamente. 
maria no constituye una política del Estado, sólo existe para niños con problemas sociales o como forma de reunir a los escolares de quinto y sexto grados en las áreas rurales dispersas, lo que posibilita que éstos al cancen una educación de mayor calidad. Los alumnos internos reciben alimentación, materiales de estudio y aseo personal, corriendo todos los gastos a cargo del Estado. La totalidad de los niños al terminar sus estudios de sexto grado tienen garantizado su ingreso a la enseñanza secundaria.

La relación alumno-profesor ascendía a 13 en 1990, pero se ha analizado la conveniencia de elevarla, dado que ello posibilitaría liberar una proporción mayor de maestros cada año para destinarlos a superarse técnica y pedagógicamente.

La proporción de escolares por profesor en la enseñanza primaria en otros países en 1990 se comportaba como sigue: U ruguay, 22\%; Argentina, 19\%; países en desarrollo de «alto desarrollo humano», 28\%; países en desarrollo de «medio desarrollo humano», 24\%.

El índice de repitencia en la enseñanza primaria es bajo, pues sólo equivale al 3\% de la matrícula, mientras ese indicador oscila al rededor del $8 \%$ entre los países en desarrollo más avanzados. O tro índice para evaluar la eficiencia de la primaria en Cuba es que el $96 \%$ de los alumnos terminan ese nivel. EI promedio de los países antes referidos es del $82 \%$. En América Latina y el Caribe, el índice de repitencia alcanza el 13\% y sólo el $45 \%$ completa la enseñanza primaria.

Un análisis breve de la información disponible sobre los docentes de la ense ñanza primaria permite la siguiente caracterización: de los 76,2 miles de maestros (en 1993), el 78\% eran mujeres; más del 50\% se habían graduado de licenciatura en Educación Primaria, y una alta proporción de los restantes cursaba esos estudios. Se ha calculado que en el año 2000 la casi totalidad del claustro poseerá ese título de nivel superior.

Los maestros primarios atienden un aula, con un promedio de carga docente directa de 22 horas semanales y el resto del tiempo (hasta 190 horas mensuales) lo ocupan en organización escolar, actividades extradocentes y superación personal).

No obstante los grandes esfuerzos desarrollados por la superación de los maestros primarios, su nivel cualitativo general es menos satisfactorio que los avances cuantitativos, debido a la masi vidad que caracterizó esa enseñanza a partir del triunfo de la Revolución, que obligó a la formación emergente de muchos de ellos, inicialmente.

La compulsión social, más que la vocación, ha estado presente en el reclutamiento de algunos. Actualmente los niveles salariales en otras actividades económicas son más gratificantes, y la conjunción de esos dos factores ha favorecido cierta movilidad de la fuerza de trabajo de este sector en los últimos años, aún cuando a nivel nacional la relación alumno-maestro es tan baja que cierta fluctuación no ocasiona alarmas, siempre que ésta sea escalonada.

EI plan de estudio de la enseñanza primaria y de la secundaria básica (grados séptimo, octavo y noveno) es único para todo el país, y todas las asignaturas son obligatorias. Los programas, textos y orientaciones metodológicas son 
elaborados por equipos de especialistas del M inisterio de Educación, con la colaboración de los docentes más experimentados, y se analizan periódicamente para introducir los cambios que garanticen su perfeccionamiento continuo.

En el primer ciclo (primero a cuarto grado) se incluyen juegos, la enseñanza integrada de la lectura y el español (lengua materna) y se brinda especial atención al desarrollo de habilidades de expresión oral, escrita y cálculos. Asimismo, se les brinda a los niños nociones del medio natural y social que los circunda.

En el segundo ciclo se priorizan las habilidades intelectuales generales. Se incluyen ciencias naturales; historia y geografía de C uba; estética (música, plástica) y educación cívica.

En la secundaria básica se inicia el estudio sistámico de la biología, la física, la química, la literatura y elementos de dibujo. Se complementa la formación de los alumnos con la educación física, artística y laboral, prestando especial atención al trabajo independiente y a las normas de conducta y convivencia social.

Los maestros y profesores tienen libertad para utilizar los métodos de enseñanza y educativos que consideren más convenientes de acuerdo a los objetivos de los programas y las características de sus educandos. También pueden adaptar las propuestas de dosificación y el orden de los contenidos incluidos en los programas de estudio.

En la secundaria básica se ocupan casi 40.000 profesores, de los cuales el $87 \%$ son licenciados (graduados de nivel superior) y el resto se encuentra estudiando para lograr su titulación universitaria. El 65\% del claustro está integrado por mujeres.

Los alumnos acceden a este nivel automáticamente, al concluir el sexto grado, y pueden optar por un status externo (secundarias urbanas) o interno (becas en el campo).

En 1993 la matrícula en secundaria básica ascendía a 350,8 miles de estudiantes ( $50 \%$ del sexo femenino), en edades entre 12 y 15 años por lo general. Al concluir este nivel, los egresados pueden seleccionar estudios en institutos preuniversitarios o en centros politécnicos, mediante un proceso escalafonario en base a las calificaciones obtenidas, los requerimientos territoriales y las capacidades instaladas.

Los profesores de secundaria tienen una carga docente entre 12 y 20 horas semanales directas y un máximo de 260 alumnos. Completan su horario laboral de forma semejante a lo explicado para los maestros de primaria. El salario puede variar en un determinado rango, en función de los resultados de la evaluación anual de su trabajo, pero el nivel básico es similar al de los restantes profesionales universitarios. Ellos, al igual que todos los trabajadores docentes, reciben modestos incrementos salariales por concepto de antigüedad cada 5 años, hasta los 25 años de trabajo.

La base material de estudio en el subsistema general básico (textos y materiales de enseñanza) se ofrece gratuitamente a los estudiantes, de acuerdo a las disponibilidades existentes en cada curso. 
Enseñanza media

Antes del triunfo de la Revolución Cubana sólo 88.000 jóvenes asistían a centros de nivel medio, y la matrícula de la enseñanza técnica y profesional no sobrepasaba los 15.000 alumnos. Se han creado las condiciones necesarias y se han ofrecido las oportunidades para asimilar en la actualidad cifras mucho mayores, aún cuando la estructura etárea de la población no demanda ahora esfuerzos en esos niveles tan intensos como en el segundo quinquenio de la década pasada (ver tabla 5).

En el año 1993 la matrícula de nivel medio ascendió a 404.000 estudiantes, de los cuales el $51 \%$ eran mujeres. La estructura de alumnos en ese nivel se muestra en la tabla 6.

Puede inferirse de la información precedente que el mayor peso de la matrícula en este nivel está en la enseñanza técnica y profesional. Esto responde a la importancia que se viene concediendo desde hace algunos años a la formación de técnicos medios, como complemento del alto número de profesionales que ya posee el país.

Recientemente ha crecido el grupo de estudiantes que accede a esta enseñanza con doceavo grado, lo que posibilita lograr un egresado con mayor preparación y más elevado nivel cultural.

Las escuelas normales están en proceso de extinción, debido a que la formación de educadoras y maestros primarios ha pasado a los institutos pedagógicos, de nivel superior. La matrícula que se registra en el año 1993 se refiere a 4 centros que desarrollan planes en liquidación y a los que preparan profesores de educación física.

Tabla 5. M atrícula en la educación media de preuniversitario y enseñanza técnica profesional.

\begin{tabular}{lrrrrr}
\hline & \multicolumn{2}{l}{ Preuniversitario } & \multicolumn{2}{c}{ Enseñanza técnica y profesional } \\
\cline { 2 - 3 } \cline { 5 - 5 } Años escolares & Total $(*)$ & Femenina & & Total & Femenina \\
\hline $1960-1961$ & 18.697 & 9.722 & 27.410 & 12.335 \\
$1970-1971$ & 15.461 & 8.040 & 27.566 & 12.405 \\
$1975-1976$ & 37.672 & 19.221 & & 114.653 & 51.594 \\
$1980-1981$ & 159.671 & 85.023 & 228.487 & 104.450 \\
$1985-1986$ & 171.661 & 101.595 & 307.129 & 138.602 \\
$1987-1988$ & 204.490 & 124.959 & 334.788 & 153.032 \\
$1989-1990$ & 236.113 & 144.511 & & 311.964 & 145.284 \\
$1991-1992$ & 195.163 & 124.468 & 293.408 & 140.916 \\
$1992-1993$ & 148.731 & 97.887 & 284.832 & 134.487 \\
$1993-1994$ & 108.386 & 72.687 & 259.355 & 120.521
\end{tabular}

Fuente: M IN ED y 0 EI para la Educación, la Ciencia y la Cultura: «Sistema Educativo N acional de Cuba», 1995. Cuadros 4, 5 y 7 del anexo estadístico.

Notas: $(*)$ Incluye 15 escuelas de iniciación deportiva (EIDE) y 15 preuniversitarias vocacionales de ciencias exactas. 
Tabla 6. Alumnos de la enseñanza media, en 1993.

\begin{tabular}{lrrr}
\hline Enseñanza & \multicolumn{1}{c}{ Total } & M ujeres & \multicolumn{1}{c}{$\%$} \\
\hline Preuniversitaria & 108.400 & 72.700 & 27 \\
Técnica y profesional & 259.300 & 120.500 & 64 \\
Escuela de oficios & 29.000 & 8.400 & 7 \\
Escuelas normales & 7.300 & 5.900 & 2 \\
Totales & 404.000 & 207.500 & 100 \\
\hline
\end{tabular}

Fuente: M IN ED y 0 EI para la Educación, la Ciencia y la Cultura: Sistema Educativo $N$ acional de Cuba, 1995. Tabla 4.1 y cuadros 4, 5, 6 y 7 del anexo estadístico.

Para atender la enseñanza media existían en Cuba, en el curso 1993-1994, 318 institutos preuniversitarios y 609 tecnológicos. Estas cifras contrastan con los 21 y los 40 centros de esas características que había en 1959. Adicionalmente hoy se dispone de 173 escuelas de oficio.

Los institutos preuniversarios pueden ser urbanos (para alumnos externos), en el campo (para estudiantes internos), vocacionales de ciencias y vocacionales militares. A éstos dos últimos acceden los estudiantes egresados de secundaria básica mediante proceso de selección que toma en cuenta el promedio alcanzado en ese nivel y los resultados de un examen de ingreso. En estos centros se refuerzan los conocimientos de las ciencias básicas y la orientación profesional.

Los jóvenes que ingresan al preuniversitario oscilan entre 15 y 16 años por lo general, y después de cursar los tres años de ese nivel, pueden continuar estudios universitarios, o en centros politécnicos medios superiores, o incorporarse a la vida social.

La educación técnica y profesional tiene la responsabilidad de formar la fuerza de trabajo calificada, de nivel medio, que requiere el desarrollo económico del país. Su estructura comprende dos niveles de formación: Ios obreros calificados y los técnicos medios.

La formación de los obreros calificados y los técnicos medios garantiza la adquisición de los conocimientos culturales y técnicos, así como los hábitos y las habilidades que requiere cada especialidad.

El nivel de ingreso a estos dos tipos de formación es de noveno y doceavo grado. La duración de los estudios varía entre 2,5 y 4 años, dependiendo de la escolaridad inicial y la complejidad de la especialidad. D espués de graduarse, los técnicos pueden incorporarse a la producción o a los servicios y, desde su puesto de trabajo, continuar estudios de más alta calificación.

Las principales especialidades de nivel medio superior (más de 70) están vinculadas a las industrias azucarera, química, alimenticia, electrónica, construcción de maquinarias, energética, producción agropecuaria, economía, geología, minería, metalurgia, transporte, comunicaciones y construcciones.

Por su parte, las de nivel medio básico (unas 17) se asocian a las ramas agropecuaria, construcción de maquinarias, construcción, transporte, energé tica, geología, minería y metalurgia. 
Este subsistema atiende los cursos de las escuelas de oficios, que tienen el objetivo de elevar la escolaridad de los jóvenes de 13 a 16 años, fundamentalmente aquellos que presentan retraso escolar o que se encuentran desvinculados del sistema nacional de educación. En estos centros se conjuga el estudio con la práctica en talleres polivalentes y posibilitan la calificación para trabajos con maderas, metales, electricidad, construcciones, costura, etc.

Corresponde también a este subsistema la atención metodológica a los cursos de capacitación técnica que desarrollan los organismos de la administración central del Estado. Esta educación cuenta con grandes talleres y laboratorios para las prácticas docentes, afectadas seriamente desde 1990 por las carencias derivadas de las limitaciones económicas que afronta el país.

Los elementos centrales que integran el plan de estudios de preuniversitario son las asignaturas de ciencias naturales, ciencias sociales, matemáticas, computación y español-literatura. Por su parte, los programas de los centros tecnológicos parten del perfil ocupacional e incluyen los conocimientos, las habilidades, las cualidades y las convicciones que debe dominar el egresado para desempeñarse adecuadamente en su actividad laboral. Todos los programas de la enseñanza media son elaborados nacionalmente, y los colectivos de asignatura de cada centro sólo pueden modificar su dosificación, métodos o medios impartidos y la concepción de las prácticas.

El claustro del subsistema de enseñanza media asciende a unos 50.000 profesores, de los cuales el 39\% son mujeres. La mayor parte de este personal docente posee nivel superior, y una alta proporción de los no titulados se encuentran estudiando en centros universitarios.

Para poder evaluar los resultados de la enseñanza media en Cuba hay que tener en consideración que ese nivel concentra el 32\% de la matrícula total del sistema y el $36 \%$ de los profesores ${ }^{6}$. La proporción de matriculados con relación al total de población que pertenece al grupo de edades correspondiente a dicho nivel asciende al $90 \%$. Entre los países en desarrollo clasificados de «alto desarrollo humano», solamente Bahamas lo supera (93\%); Chipre Io iguala (90\%) y se acercan Barbados (89\%), República de Corea (87\%), Trinidad-Tobago $(84 \%)$ y U ruguay $(81 \%)$.

El índice de repitencia en la enseñanza secundaria en Cuba es relativamente satisfactorio (3\%) si se compara con el de los países en desarrollo más avanza$\operatorname{dos}(5 \%)$.

Los principales retos de este subsistema de educación en Cuba están asociados a elementos cual itativos. D e una parte los preuniversitarios tienen que elevar la preparación de los egresados para que éstos puedan satisfacer los requerimientos del ingreso a la educación superior, que son cada vez más exigentes como consecuencia del perfeccionamiento de los planes de estudio a ese nivel, en correspondencia con el progreso científico técnico. Ello implica modificar

6. Se ha sumado a los datos de preuniversitario, tecnológico, escuelas normales y de oficio, los correspondientes a la secundaria básica, a los efectos de facilitar la comparación con otros países en desarrollo, que consideran el nivel secundario a partir del séptimo grado. 
los métodos de enseñanza, avanzar en la actualización científica de los docentes y ampliar la base bibliográfica disponible.

D e otra parte, en la enseñanza técnica se ha ido produciendo una obsolescencia física y moral en los talleres, equipos y herramientas requeridos por el proceso docente, como consecuencia de las dificultades económicas por las que atraviesa el país. Adicionalmente hay escasez de materiales, no sólo en los centros docentes, sino también en las entidades laborales donde los alumnos podrían realizar sus prácticas, y ello afecta al desarrollo de habilidades, tan importante en este tipo de enseñanza.

A lo antes expuesto hay que sumar la incertidumbre para poder garantizar ubicación laboral a los egresados del nivel medio, como consecuencia de la contracción de las actividades en al gunos sectores, factor que actúa negativamente en la motivación de los jóvenes estudiantes.

La superación de estas dificultades no sólo demanda una esmerada atención gubernamental y de los centros docentes, sino que depende en buena medida del proceso de recuperación económica del país.

Será necesario crear una dinámica de aprovechamiento máximo de las instalaciones y áreas de campo a disposición de la enseñanza media para que en el período de aprendizaje práctico de los alumnos se aporten los máximos bene ficios posibles a la economía nacional en producciones agropecuarias, piezas de respuestos, artículos de consumo doméstico artesanales, ensamblaje de equipos, etc., y con la utilización de una parte de esos resultados se facilite el mejoramiento material de este nivel de educación.

\section{Enseñanza superior}

Actualmente existen 57 centros de educación superior entre los 124 institutos pedagógicos, 15 de ciencias médicas, 8 escuelas superiores militares y 15 centros universitarios. En estas instituciones se cursan 14 carreras técnicas, 10 deCiencias $N$ atuales y M atemáticas, 3 de Ciencias M édicas, 4 de Agropecuaria, 2 de Economía, 13 de Ciencias Sociales, 15 de Pegagogía, 6 deArtey 1 de Cultura Física.

Esta forma de enseñanza ha sufrido importantes transformaciones durante el período revolucionario. En la actual idad la matrícula es varias decenas de veces superior a la de 1957, y ascendía en 1993 a 166.000 estudiantes de pregrado, que representaban el $7 \%$ del total de alumnos del sistema educacional. Inmediatamente resalta la desproporción que ya se está presentando entre losniveles de egresados de la enseñanza media y las posibilidades de ingreso al nivel superior.

$\mathrm{H}$ ay que tener en consideración que todos los estudiantes egresados de los cursos diurnos (no trabajadores) tienen garantizada su ubicación en una plaza laboral, en correspondencia con su perfil, por lo que los niveles de ingreso tie nen que adecuarse a las posibilidades de empleo, y éstas a su vez dependen de las necesidades del país en cada esfera concreta.

El hecho de que en períodos anteriores a 1988 no se requiriera examen de ingreso a la educación superior, y las limitaciones para el acceso a ese nivel fue- 
ran prácticamente inexistentes, determinó graduaciones masivas en casi todas las especialidades, que contribuyó a una relativa saturación en muchas de ellas, respecto a las posibilidades de plazas adicionamos la contracción de la economía que se ha venido produciendo en la presente década, resulta fácil comprender que no se pueda asegurar empleos ilimitados anualmente a graduados universitarios.

La actual contradicción podría atenuarse si se flexibiliza el compromiso de garantizar empleo a todos los graduados, ya que a pesar de las limitaciones económicas del país, que no han dejado de repercutir considerablemente en la educación superior, ésta tiene potencialidades para asimilar matrículas mayores. Pero ése es un tema de política educacional, que compete decidir a los niveles gubernamentales.

D e cual quier forma, es difícil que no se presenten nuevamente niveles tan elevados de matrícula como los alcanzados en la década de 1980, cuando se llegó al orden de los 40.000 egresados anualmente, porque en ese período era muy alta la proporción de estudiantes trabajadores, pero ya en el seno de éstos la mayoría de los que aspiraban a profesionales se han graduado o han sobrepasado la edad permisible para ingresar a las universidades, y sólo los jóvenes que se van incorporando a la vida laboral procedentes de la enseñanza media serian cantera de los cursos para trabajadores.

C iertamente esta modalidad de enseñanza es una oportunidad más que se ofrece a la población para cursar estudios superiores, y se complementa con la enseñanza dirigida, a la cual se accede sin restricciones, pero que tiene un régimen de estudios por cuenta del alumno, con idéntico plan y rigor que los cursos regulares. Ya ascienden a varios cientos los graduados universitarios de esta modalidad.

Acceso a la educación superior: El plan de ingreso para los cursos diurnos se elabora anualmente, y se publica la cantidad de plazas disponibles para cada especialidad (éstas se determinan en base a las necesidades del país de fuerza de trabajo calificada de nivel superior). La asignación de plazas se realiza en base a un escalafón confeccionado, en el que se considera el índice académico del nivel precedente y la puntuación alcanzada en el examen de ingreso. Para al gunas carreras se establecen requisitos complementarios. Tienen derecho a optar por este procedimiento todos los egresados de nivel medio superior, con idénticas oportunidades individuales.

El sistema de admisión a los cursos para trabajadores se basa en un ordenamiento escalafonario que toma en cuenta los resultados de un examen de ingreso sobre matemática y español, además de los requisitos de edad (menores de 35) y vínculo laboral activo.

Para acceder a la enseñanza dirigida (o libre) sólo se requiere tener el nivel de enseñanza media superior, al igual que para presentarse a los exámenes de concurso que anualmente se convocan para una cuota reducida de plazas en ciertas especialidades, y que brinda una opción más a los que intentaron el ingreso en oportunidades anteriores sin éxito.

Las medidas adoptadas para regular el ingreso a la educación superior han 
tenido una significativa repercusión en la calidad y en la eficiencia del proceso docente educativo. Los alumnos entran cada vez con mejor preparación y motivación, aunque todavía con grandes limitaciones. En la estructura de la matrícula se destaca que el $58 \%$ son mujeres, pero en los cursos diurnos hay especialidades donde la proporción de éstas es muy elevada.

También es significativa la presencia femenina en el claustro universitario, y asciende al $46 \%$ de los casi 25.000 docentes activos en 1993. La relación alumno-profesor es baja si sólo se toman en consideración los estudiantes de pregrado (7), pero en los últimos años ha ido ganando importancia paulatina la actividad de postgrado en la educación superior debido a que el país cuenta con más de medio millón de egresados universitarios que tienen que ser atendidos para garantizar su recalificación y actualización continua, en diversas modalidades.

Las principales variantes que adopta el postgrado son cursos, entrenamientos, estudios, diplomado, especialidad, maestría y doctorado. Este cuarto nivel de enseñanza en algunos centros universitarios tiene una matrícula 405 veces mayor que la de pregrado.

0 tro componente importante de la educación superior en Cuba es la actividad científico-investigativa, que se vincula a los principales proyectos del desarrollo económico y social del país en las diferentes ramas del saber. La contribución que realizan los docentes universitarios a la investigación y la solución de importantes problemas de corto o largo plazo es muy destacada y reconocida en la actualidad. No obstante, el vínculo con la producción y los servicios está aún por debajo de las potencialidades existentes, y ésta es una dirección que demanda mayores avances para contribuir a la acelerada aplicación de los resultados científicos y técnicos.

Lo antes expuesto se vincula con la necesidad de continuar reforzando la integración docencia-investigación-actividad laboral en la formación de los estudiantes y el desarrollo del trabajo transdisciplinario, que son dos premisas para el perfeccionamiento de los planes de estudio que requiere el graduado del próximo siglo.

Planes de estudio: La educación superior actual mente atiende 78 carreras de perfil amplio, con planes de estudios entre 5.000 y 6.000 horas, y duración de 5 años (excepto M edicina, que son 6), con un fuerte componente laboral.

Los planes son elaborados por comisiones nacionales de especialistas y constituyen documentos rectores para todos los centros, pero cada vez más se introducen elementos de flexibilidad en el manejo de los mismos, en función de la especificidad territorial y las características de los alumnos. Los planes incluyen los programas sintéticos de las asignaturas con sus contenidos esenciales, pero cada colectivo de profesores elabora el programa analítico. Actualmente se ha superado la etapa de rigidez y la uniformidad metodológica que caracte rizó el período de matrículas muy numerosas y claustros insuficientemente pre parados.

Los elementos antes expuestos de mayor libertad individual en el ejercicio de la docencia y una alta participación en actividades científicas, ha elevado 
el nivel de satisfacción del claustro universitario, independientemente de que sus niveles de remuneración económica son inferiores a los de otros sectores priorizados por el país o de trabajadores ocupados en actividades informales. D ebe resaltarse que, comparativamente con otros profesionales que se desempeñan en el sector público, éste es el segmento de más altos salarios en Cuba.

\section{Consideraciones finales}

La estrategia cubana de desarrollo ha concedido especial atención al sistema educativo como uno de los ejes para lograr la modernización de la estructura económica, sin grandes desigualdades sociales. Los planes educacionales no han estado exentos de errores e insuficiencias, pero han logrado resultados objetivos que sitúan al país en una decorosa posición mundial por sus índices de alfabetización, maestros per cápita, grado de escolarización y proporción de profesionales y técnicos, entre otros.

El análisis de al gunos indicadores sociológicos carece de mucho sentido en Cuba para evaluar el proceso de educación, porque las oportunidades para acceder a la enseñanza están disponibles para la totalidad de los ciudadanos, no formal, sino realmente. D e ahí que en el ciclo básico (hasta noveno grado) las diferenciaciones raciales, por sexo, edades o procedencia social responden fundamental mente a las características de la población en cada momento porque la matrícula abarca al $99 \%$ de los posibles alumnos. Sólo en los niveles medio, superior y universitario algunos matices podrían resultar de interés en estos momentos, cuando el acceso universitario está sometido a un proceso escalafonario basado en el promedio al canzado en el nivel precedente y el resultado del examen de ingreso. Lamentablemente no está registrada la información estadística para real izar esas val oraciones.

Aun cuando los principales logros educacionales se han preservado en los últimos años, la calidad del proceso docente educativo se ha visto afectada debido a la difícil situación económica por la que atraviesa el país desde 1989, especialmente por la disminución del proceso inversionista para el sector; el insuficiente mantenimiento; la escasez de medios, materiales, reactivos y equipos para la enseñanza; la obsolescencia de talleres y maquinarias para el subsistema tecnológico; la no sistemática actualización de bibliografía e información internacional; las dificultades para completar los requerimientos de computación; las limitaciones para impresión y edición de literatura especializada, etc.

Es imprescindible mejorar la eficiencia económica de las actividades educacionales, aprovechando al máximo los recursos disponibles, reduciendo los costos unitarios, elevando los ingresos que puede generar el propio sector, incrementando las actividades autofinanciadas, y por otras vías, que posibiliten ade cuar la proporción de gastos públicos del sector a las posibilidades de la economía cubana y ajustar las expectativas educacionales al desarrollo objetivo alcanzable por el país.

D ado que en lo esencial están ya resueltas las principales deformaciones e insuficiencias heredadas por la Revolución de la república neocolonial en el 
sector educacional, se debe poner el énfasis en el perfeccionamiento de los aspectos cualitativos del sistema, a través de la modernización continua de los planes y programas de estudios, en correspondencia con las necesidades del progreso científico-técnico; la superación sistemática del personal docente; la transformación de los métodos de enseñanza para favorecer cada vez más la polémica, el desarrollo de la creatividad y de habilidades prácticas, la independencia de los estudiantes, etc.

Partiendo de los niveles ya logrados de cal ificación general de la población cubana se debe analizar la conveniencia de destinar mayores esfuerzos en el futuro hacia la recalificación y la actualización de la fuerza de trabajo en especializaciones concretas para elevar la eficiencia laboral.

Las instituciones educacionales cubanas deben continuar mejorando su trabajo de extensión cultural hacia la comunidad, vinculando más a sus docentes y estudiantes al proceso de diagnóstico y búsqueda de soluciones de los principales problemas educacionales y generales del territorio o la nación. Con métodos participativos se pueden lograr interesantes y útiles análisis sobre temas tan problemáticos como el ingreso a la educación superior; la reducción de matrículas en los preuniversitarios urbanos; el sistema de estímulos a maestros y profesores; la dialéctica uniformidad-flexibilidad en el ejercicio de las cátedras; la correspondencia entre recursos disponibles y calidad de la educación, y otros.

Resulta imprescindible el mejoramiento de los sistemas organizacionales de los centros educativos, garantizando mayor autonomía a éstos y el reforzamiento de la autoridad de los claustros a todos los niveles, con el objetivo de que estas instituciones se conviertan en ejemplos de una cultura organizacional basada en los principios y en las normas de conducta que deben guiar la formación de los educandos. 